\title{
Expressional analysis of the astrocytic Kir4.1 channel in a pilocarpine-induced temporal lobe epilepsy model
}

\author{
Yuki Nagao ${ }^{\dagger}$, Yuya Harada ${ }^{\dagger}$, Takahiro Mukai, Saki Shimizu, Aoi Okuda, Megumi Fujimoto, Asuka Ono, \\ Yoshihisa Sakagami and Yukihiro Ohno*
}

Laboratory of Pharmacology, Osaka University of Pharmaceutical Sciences, Osaka, Japan

\section{Edited by:}

Roberto Di Maio, University of

Pittsburgh, USA

Reviewed by:

Maarten H. P. Kole, Netherlands

Institute for Neuroscience,

Netherlands

Ithai Rabinowitch, Hebrew University

of Jerusalem, Israel

\section{${ }^{*}$ Correspondence:}

Yukihiro Ohno, Laboratory of

Pharmacology, Osaka University of

Pharmaceutical Sciences, 4-20-1

Nasahara, Takatsuki, Osaka 569-1094,

Japan

e-mail:yohno@gly.oups.ac.jp

'Yuki Nagao and Yuya Harada have contributed equally to this work.

The inwardly rectifying potassium (Kir) channel Kir4.1 in brain astrocytes mediates spatial $\mathrm{K}^{+}$ buffering and regulates neural activities. Recent studies have shown that loss-of-function mutations in the human gene KCNJ10 encoding Kir4.1 cause epileptic seizures, suggesting a close relationship between the Kir4.1 channel function and epileptogenesis. Here, we performed expressional analysis of Kir4.1 in a pilocarpine-induced rat model of temporal lobe epilepsy (TLE) to explore the role of Kir4.1 channels in modifying TLE epileptogenesis. Treatment of rats with pilocarpine $(350 \mathrm{mg} / \mathrm{kg}$, i.p.) induced acute status epilepticus, which subsequently caused spontaneous seizures 7-8 weeks after the pilocarpine treatment. Western blot analysis revealed that TLE rats (interictal condition) showed significantly higher levels of Kir4.1 than the control animals in the cerebral cortex, striatum, and hypothalamus. However, the expression of other Kir subunits, Kir5.1 and Kir2.1, remained unaltered. Immunohistochemical analysis illustrated that Kir4.1-immunoreactivity-positive astrocytes in the pilocarpine-induced TLE model were markedly increased in most of the brain regions examined, concomitant with an increase in the number of glial fibrillary acidic protein (GFAP)-positive astrocytes. In addition, Kir4.1 expression ratios relative to the number of astrocytes (Kir4.1-positive cells/GFAP-positive cells) were region-specifically elevated in the amygdala (i.e., medial and cortical amygdaloid nuclei) and sensory cortex. The present study demonstrated for the first time that the expression of astrocytic Kir4.1 channels was elevated in a pilocarpine-induced TLE model, especially in the amygdala, suggesting that astrocytic Kir4.1 channels play a role in modifying TLE epileptogenesis, possibly by acting as an inhibitory compensatory mechanism.

Keywords: Kir4.1 channel, astrocytes, temporal lobe epilepsy, status epilepticus, spatial potassium buffering, pilocarpine

\section{INTRODUCTION}

The spatial $\mathrm{K}^{+}$buffering by astrocytes removes excess extracellular $\mathrm{K}^{+}$at synapses and transports them into regions of low $\mathrm{K}^{+}$ concentration such as blood vessels, regulating neuronal activities (Walz, 2000; Kofuji and Newman, 2004; Simard and Nedergaard, 2004; Butt and Kalsi, 2006). The $\mathrm{K}^{+}$buffering currents are mediated by inwardly rectifying potassium (Kir) channels which are expressed in astrocytes (Tanemoto et al., 2000; Hibino et al., 2004; Kofuji and Newman, 2004; Simard and Nedergaard, 2004; Butt and Kalsi, 2006). These comprise Kir4.1 channels, homo-tetramers of Kir4.1 subunits, and Kir4.1/5.1 channels, hetero-tetramers of Kir4.1 and Kir5.1 subunits, which conduct large inward $\mathrm{K}^{+}$currents at potentials negative to $\mathrm{K}^{+}$equilibrium potential (Tanemoto et al., 2000; Ohno et al., 2007; Su et al., 2007; Furutani et al., 2009). In addition, spatial $\mathrm{K}^{+}$buffering is linked to glutamate uptake and/or aquaporin-4-mediated water transport by astrocytes (Nagelhus et al., 1999; Amiry-Moghaddam and Ottersen, 2003; Puwarawuttipanit et al., 2006; Djukic et al., 2007; Kucheryavykh et al., 2007).

Abbreviations: GFAP, glial fibrillary acidic protein; GTC, generalized tonic-clonic; Kir, inwardly-rectifying potassium; SE, status epilepticus; TLE, temporal lobe epilepsy.
Recent clinical studies have shown that mutations in the human gene KCNJ10 encoding Kir4.1 cause EAST (epilepsy, ataxia, sensorineural deafness, and tubulopathy) or SeSAME (seizures, sensorineural deafness, ataxia, mental retardation, and electrolyte imbalance) syndrome consisting of generalized tonic-clonic (GTC) seizures, ataxia, hearing loss, and abnormal renal excretion of electrolytes (Bockenhauer et al., 2009; Scholl et al., 2009). The most frequent mutation of KCNJ10 was R65P at the cytoplasmic end of transmembrane region (TM)1 and others include G77R (TM-1), C140R (extracellular loop between TM-1 and TM-2), T164I, A167V (cytoplasmic end of TM-2), R175Q, R199X, and R297C (C-terminal domain; Reichold etal., 2010; Sala-Rabanal etal., 2010; Tang etal., 2010). All these mutations caused drastic decreases in $\mathrm{K}^{+}$currents mediated by Kir4.1 and Kir4.1/5.1 channels, suggesting that the impaired functioning of astrocytic Kir4.1 channels causes epileptic seizures by disrupting spatial $\mathrm{K}^{+}$buffering. In addition, several SNPs of KCNJ10 have been shown to be associated with temporal lobe epilepsy (TLE) with febrile seizures (Heuser etal., 2010). Expressional analysis also revealed pathophysiological alterations in Kir4.1 expression in patients with TLE (Das etal., 2012; Heuser et al., 2012; Steinhäuser et al., 2012), 
suggesting a potential involvement of Kir4.1 channels in TLE epileptogenesis. However, information on the modulatory role of Kir4.1 in the generation and/or development of TLE is still very limited.

In the present study, we performed expressional analysis of Kir4.1 in a pilocarpine-induced rat model of TLE to explore the pathophysiological role of Kir4.1 channels in TLE epileptogenesis. The expressions of Kir5.1 and Kir2.1, other Kir subunits expressed in astrocytes, were also evaluated for comparison.

\section{RESULTS \\ PILOCARPINE-INDUCED TLE MODEL}

All the TLE rats $(\mathrm{N}=11)$ used herein experienced pilocarpine (350 mg/kg, i.p.)-induced status epilepticus (repeated and sustained clonic seizures) and showed spontaneous seizures (i.e., wild running/jumping and GTC seizures) 7-8 weeks after the pilocarpine treatment. The animals, which were given pilocarpine but did not experience status epileptics and any seizure activity thereafter ( $7-8$ weeks), were used as the control $(N=11)$. Four and seven animals in each group were subjected to Western blot and immunohistochemical analysis, respectively.

\section{WESTERN BLOT ANALYSIS}

As reported previously (Connors et al., 2004; Seifert et al., 2009; Harada et al., 2013), Kir4.1 was detected primarily as a tetramer $(\sim 160 \mathrm{kDa})$ in all brain regions examined in TLE and control rats (Figure 1A). Two-way ANOVA revealed no significant interaction $[F(1,60)=1.61, P=0.13]$, but significant main effects of groups $[F(1,60)=23.24, P<0.01]$ and regions $[F(9,60)=10.80$, $P<0.01]$. Expression levels of Kir4.1 were relatively high in the striatum $(\mathrm{St})$ and pons/medulla oblongata $(\mathrm{P} / \mathrm{MO})$. As compared to control animals, TLE rats showed significantly higher Kir4.1 levels in the frontal cortex (fCx, $P<0.05$ ), occipito-temporal cortex (otCx, $P<0.05)$, St $(P<0.01)$, hypothalamus $(\mathrm{Ht}$, $P<0.05)$, and $\mathrm{P} / \mathrm{MO}(P<0.01$; Figures $1 \mathbf{A}, \mathbf{B})$. These changes were region-specific and the Kir4.1 levels in other brain regions [i.e., parieto-temporal cortex ( $\mathrm{ptCx})$, hippocampus $(\mathrm{Hpc})$, thalamus (Th), midbrain (Mid), and cerebellum (Cer)] remained unaltered.

In contrast to Kir4.1, Kir5.1 and Kir2.1 subunits were detected mainly as monomers (Kir5.1: $50 \mathrm{kDa}$, Kir2.1: $45 \mathrm{kDa}$ ) in all 10 regions (Figure 1A). Levels of Kir5.1 were relatively high in the ptCx and Mid while the Kir2.1 levels were high in the Mid and low in the cerebral cortices and Cer (Figures 1C,D). Analysis of Kir5.1 expression showed only a significant main effect of regions $[F(9,60)=7.97, P<0.01]$ without a significant interaction $[F(9,60)=0.32, P=0.96]$ or a main effect of groups $[F(9,60)=0.77, P=0.38]$. Thus, no significant differences in the expression levels of Kir5.1 were observed between TLE and control rats in all 10 regions (Figure 1C). On the other hand, analysis of Kir2.1 expression revealed significant main effects of groups $[F(1,60)=7.93, P<0.01]$ and regions $[F(9,60)=13.9$, $P<0.01]$ without a significant interaction $[F(9,60)=1.24$, $P=0.29$ ]. Among 10 regions, only the Kir2.1 level in the St was significantly $(P<0.01)$ higher in TLE than in control rats (Figure 1D).

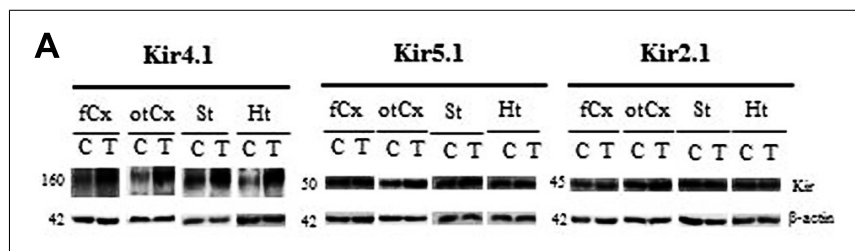

\section{B}

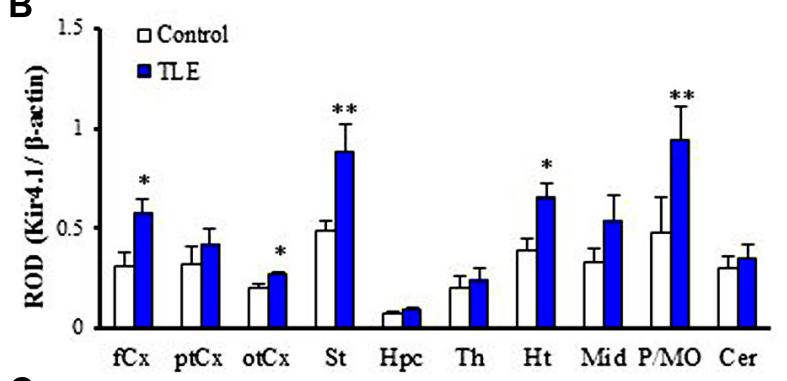

C
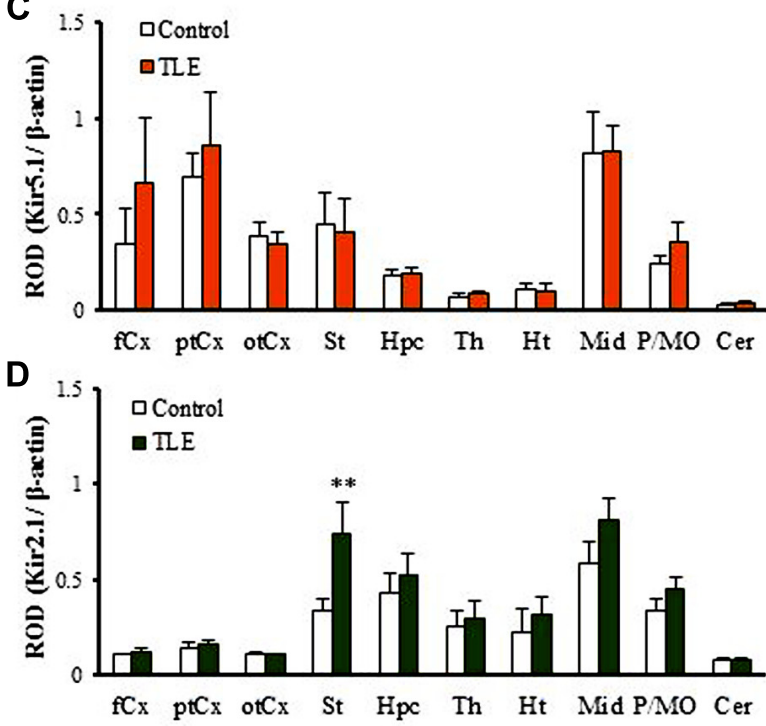

FIGURE 1 | Western blot analysis for Kir4.1, Kir5.1 and Kir2.1 expression in pilocarpine-induced TLE rats. (A) Representative Western blots visualizing Kir4.1, Kir5.1, and Kir2.1 expression in the frontal cortex (fCx), occipito-temporal cortex (otCx), striatum (St), and hypothalamus ( $\mathrm{Ht})$. (B-D) Regional expression of Kir4.1 (B), Kir5.1 (C), and Kir2.1 (D) in pilocarpine-induced TLE rats. Kir expression was expressed as relative optical density (ROD) to $\beta$-actin. $\mathrm{fCx}$, frontal cortex; ptCx, parieto-temporal cortex; otCx, occipito-temporal cortex; St, striatum; Hpc, hippocampus; Th, thalamus; Ht, hypothalamus; Mid, midbrain; $\mathrm{P} / \mathrm{MO}$, pons/medulla oblongata; Cer, cerebellum. Each column represents the mean $\pm \mathrm{SEM}$ of four animals. ${ }^{*} P<0.05,{ }^{*} P<0.01$, significantly different from the control rats

\section{IMMUNOHISTOCHEMICAL ANALYSIS FOR KIR4.1 EXPRESSION}

Since Western blot analysis revealed that pilocarpine-induced TLE rats showed elevated Kir4.1 expression in the $\mathrm{fCx}$ and otCx, we further conducted immunohistochemical analysis for Kir4.1 expression using frontal (Bregma $+1.68 \mathrm{~mm}$ level) and occipitotemporal (Bregma $-3.00 \mathrm{~mm}$ level) brain slices (Figure 2A). With regard to the expression patterns of Kir4.1-immunoreactivity (IR), we have previously shown that Kir4.1 was primarily stained in astrocytes which typically show a stellate-shape and were 


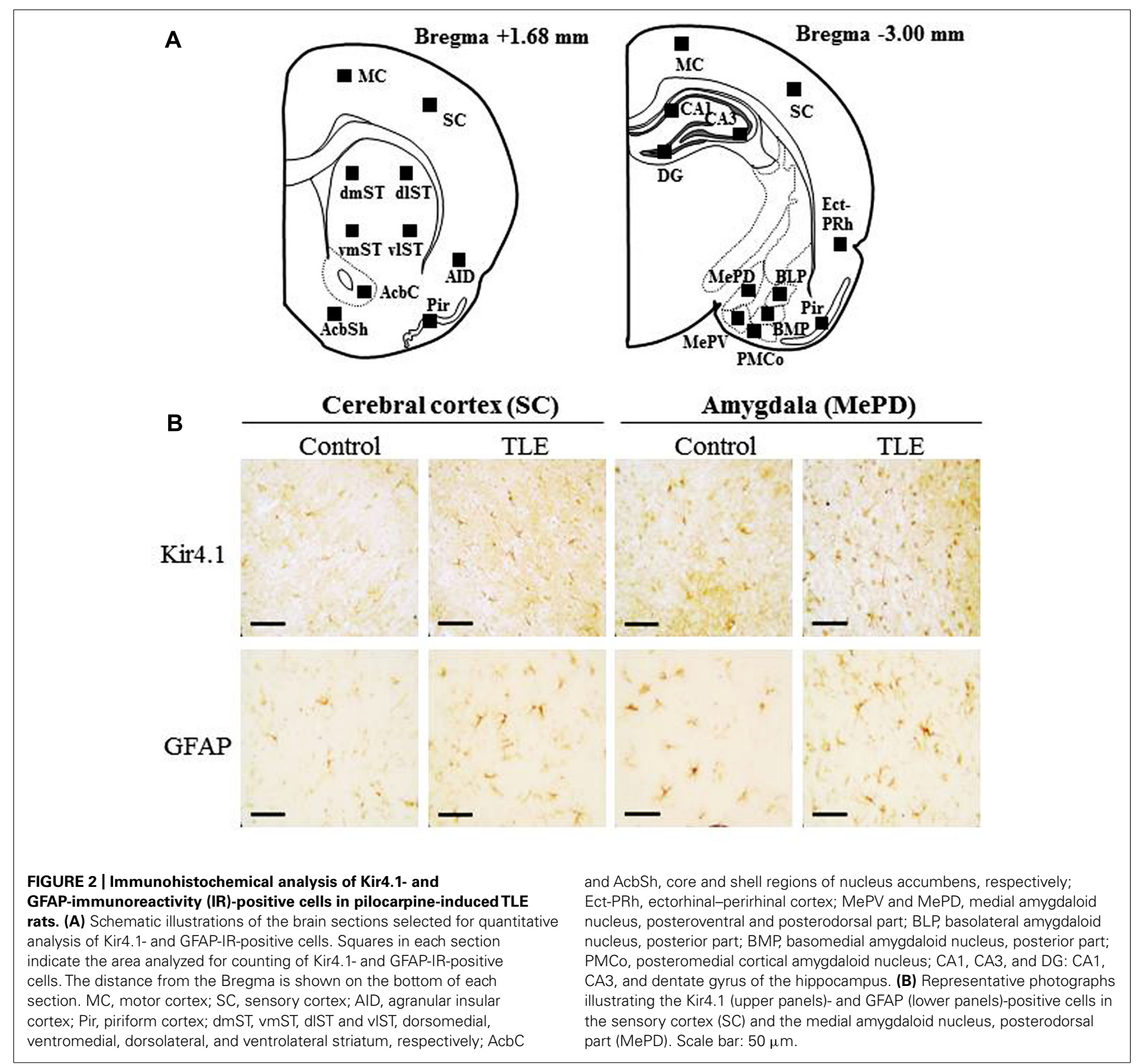

specifically co-stained with glial fibrillary acidic protein (GFAP; an astrocyte marker; Harada etal., 2013; also see Figure A1 in Appendix). Although Kir4.1-IR was also found in a small population of round-shaped (small) cells, which might possibly represent oligodendrocyte precursor cells (Maldonado et al., 2013), we omitted them from the analysis and solely counted the stellate-shaped astrocytes probe with anti-Kir4.1 antibody. In addition, to evaluate changes in the total number of astrocytes per se and the Kir4.1 expression ratio relative to the total number of astrocytes, we also performed immunohistochemical analysis of GFAP using paired successive slices obtained from the same animal.

In accordance with previous studies (Connors et al., 2004; Seifert etal., 2009; Harada etal., 2013), Kir4.1 was mostly expressed in stellate-shaped cells (Figure 2B). Two-way ANOVA revealed significant interaction groups $\times$ regions $[F(21$, $264)=1.91, P<0.05]$ and significant main effects of groups $[F(1,264)=410.45, P<0.01]$ and regions $[F(21,264)=3.50$, $P<0.01]$. In pilocarpine-induced TLE rats, Kir4.1 expression was significantly elevated in all brain regions examined [dentate gyrus of the Hpc (DG) and dorsomedial St (dmST): $P<0.05$, other regions: $P<0.01$ ] except for the agranular insular cortex dorsal part (AID; Figures 2-4). The number of Kir4.1-IR-positive astrocytes increased two to four times the control levels in TLE animals and these changes were prominent in the sensory cortex (SC), lateral St, and amygdala (Figures $3 \mathrm{~A}$ and $\mathbf{4 A}$ ). In addition, the number of GFAP-IR-positive astrocytes per se also increased in pilocarpine-induced TLE rats (Figures 3B and 4B). Analysis 
of GFAP expression showed significant main effects of groups $[F(1,264)=333.16, P<0.01]$ and regions $[F(21,264)=6.26$, $P<0.01]$ without a significant interaction $[F(1,264)=0.86$, $P=0.65]$. The numbers of GFAP-IR-positive cells in all 22 brain regions examined were significantly [piriform cortex $(\mathrm{Pir}) \mathrm{B}+1.68$ : $P<0.05$, other regions: $P<0.01]$ higher in TLE than in control rats. We then compared the Kir4.1 expression ratios relative to the number of astrocytes (Kir4.1-IR-positive cells/GFAP-IRpositive cells). Two-way ANOVA revealed significant interaction $[F(21,264)=1.78, P<0.05]$ and significant main effects of groups $[F(1,264)=12.82, P<0.01]$ and regions $[F(21$, $264)=2.36, P<0.01]$. The relative Kir4.1 expression ratios in astrocytes were $0.3-0.8$ in most regions of the brain in the control animals $(0.557 \pm 0.022)$, but the values were significantly $(P<0.01)$ increased in TLE group $(0.652 \pm 0.019)$. These changes were region-specific and significant increases were observed in the posteroventral $(\mathrm{MePV}, P<0.05)$ and posterodorsal $(\mathrm{MePD}$, $P<0.01)$ parts of the medial amygdaloid nucleus, the posteromedial cortical amygdaloid nucleus (PMCo, $P<0.05$ ), ventrolateal St (vlST, $P<0.05)$, SC $(P<0.01)$, and Pir $(P<0.05$; Figures $3 \mathrm{C}$ and $4 \mathrm{C})$.

\section{DISCUSSION}

Temporal lobe epilepsy is the most common type of partial complex seizure in adulthood (Hauser et al., 1996; Wieser, 2004). The main features of TLE include (1) localization of seizure foci in the limbic structures (e.g., Hpc and amygdala), (2) existence of a "latent period," a seizure-free time interval following the initial precipitating injury, (3) incidence of mesial sclerosis leading to atrophy (e.g., neuronal loss and gliosis) in the limbic structures (Mathern et al., 1997; Bartolomei et al., 2005; Curia et al., 2008). The pilocarpine-induced TLE model shares important features of human TLE such as (1) presence of a latent period followed by spontaneous recurrent seizures, (2) occurrence of wide spread brain injuries resembling human TLE, (3) similarity of drug responses to human TLE (e.g., relatively resistant to conventional antiepileptics; Leite et al., 1990; Cavalheiro et al., 1991; Glien et al., 2002; Löscher, 2002; Wieser, 2004; Chakir et al., 2006; Curia et al., 2008). The present study demonstrated for the first time that expression of astrocytic Kir4.1 channels mediating spatial $\mathrm{K}^{+}$buffering was markedly elevated in a pilocarpine-induced TLE model. The elevation of Kir4.1 expression in the TLE model was characterized by the following points, (1) subunit-specificity for Kir4.1, (2) a partial association with an increase in the number of astrocytes (i.e., astrogliosis) and (3) the most prominent elevation in the amygdala.

In this study, Western blot analysis revealed that the pilocarpine-induced TLE model exhibits a subunit-specific increase in the Kir4.1 expression with negligibly affecting the level of Kir5.1 and Kir2.1 subunits. Kir5.1 subunits, like Kir4.1, are expressed in astrocytes and form heteromeric Kir4.1/5.1 channels with Kir4.1, mediating $\mathrm{K}^{+}$buffering (Tanemoto et al., 2000; Hibino etal., 2004; Kofuji and Newman, 2004). In contrast, Kir2.1 subunits are predominantly expressed in neurons to regulate the resting membrane potential while several reports show that astrocytes also express Kir2.1 to some degree in several brain regions (e.g., Pir and olfactory bulb; Howe et al., 2008; Kang et al.,

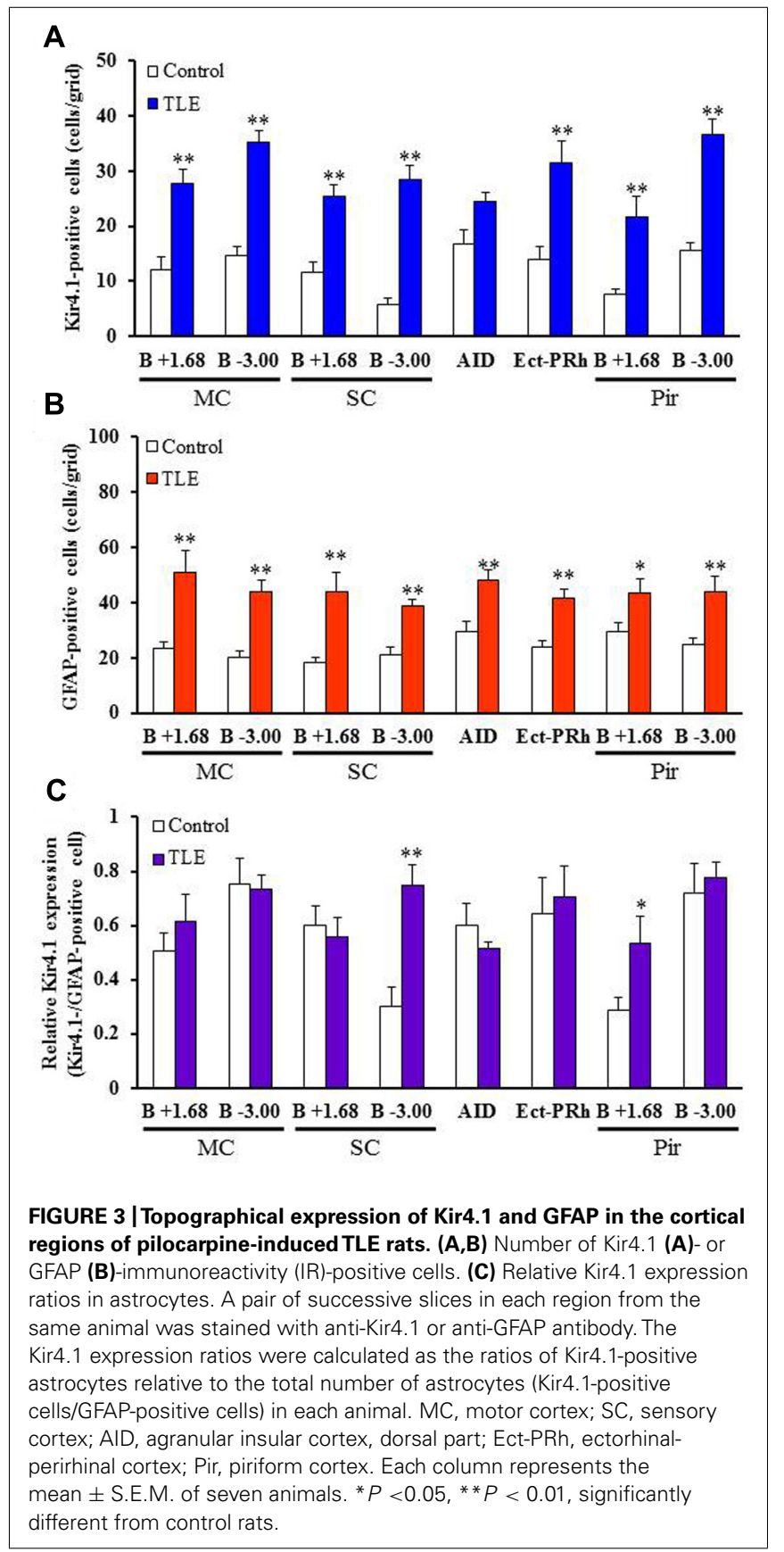

2008). Our results suggest that, among astrocytic Kir channels, Kir4.1 channels play the most important role in modulating TLE epileptogenesis.

Elevation of Kir4.1 expression in the pilocarpine-induced TLE model was widely spread throughout brain regions examined and these changes were generally associated with an increase in the number of astrocytes, which was probably due to astrogliosis following status epilepticus-induced brain injury (Leite et al., 1990; Cavalheiro etal., 1991; Borges et al., 2003; Curia et al., 2008). Although astrogliosis may also contribute to epileptogenesis, it can compensate abnormal discharges and promote tissue repair. Astrocytes can reduce abnormal neural excitation 


\section{A}

B
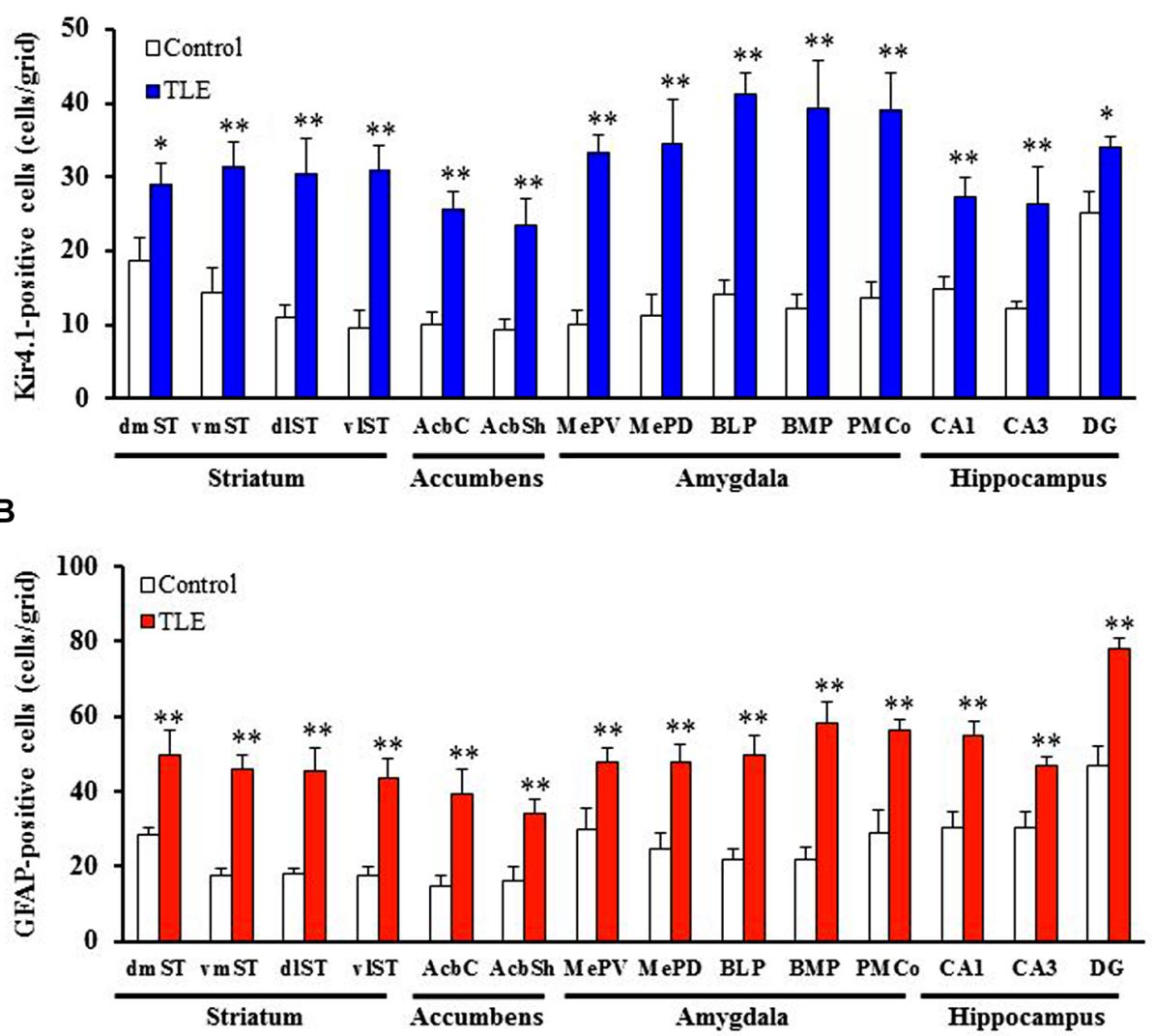

C

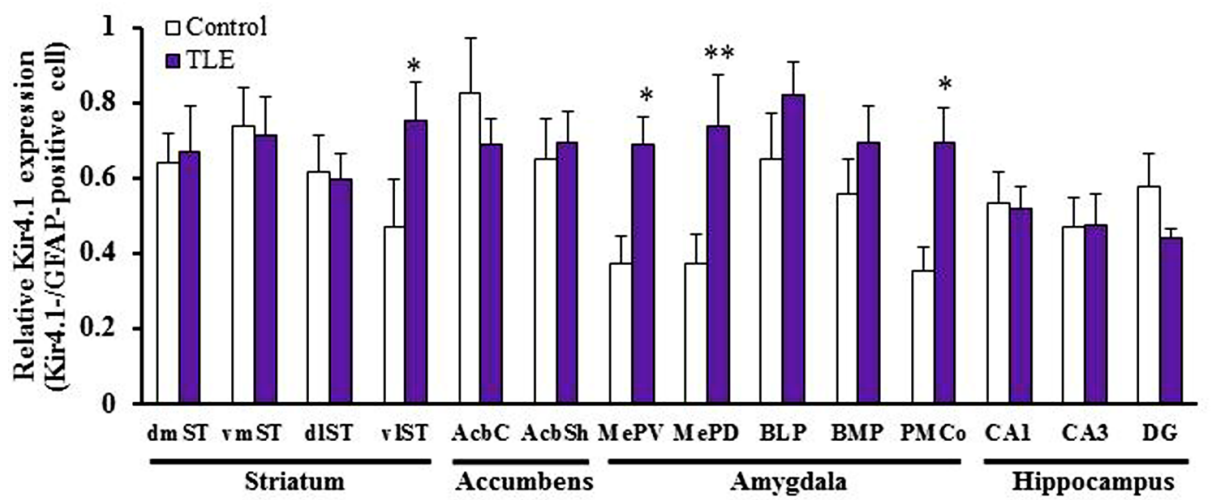

FIGURE 4 | Topographical expression of Kir4.1 and GFAP in the basal ganglia and limbic regions of pilocarpine-induced TLE rats. (A,B) Number of Kir4.1 (A)- or GFAP (B)-immunoreactivity (IR)-positive cells. (C) Relative Kir4.1 expression ratios in astrocytes. A pair of successive slices in each region from the same animal was stained with anti-Kir4.1 or anti-GFAP antibody. The Kir4.1 expression ratios were calculated as the ratios of Kir4.1-positive astrocytes relative to the total number of astrocytes (Kir4.1 Kir4.1-positive cells/GFAP-positive cells) in each animal. dmST, vmST,
dIST, and vIST, dorsomedial, ventromedial, dorsolateral, and ventrolateral striatum, respectively; $A c b C$ and $A c b S h$, core and shell regions of the nucleus accumbens, respectively; MePV and MePD, medial amygdaloid nucleus, posteroventral and posterodorsal part; BLP, basolateral amygdaloid nucleus, posterior part; BMP, basomedial amygdaloid nucleus, posterior part; $\mathrm{PMCo}$ posteromedial cortical amygdaloid nucleus; CA1, CA3, and DG, CA1, CA3, and dentate gyrus of the hippocampus. Each column represents the mean \pm SEM of seven animals. ${ }^{*} P<0.05,{ }^{*} P 0.01$, significantly different from control rats. by spatial buffering of potassium and by taking up synaptically released glutamate. In addition, they can secrete growth factors [e.g., glial cell line-derived neurotrophic factor (GDNF) and nerve growth factor (NGF)] and cytokines (e.g., TNF- $\alpha$ ) that mediate neuronal survival, axonal/dendritic sprouting, and homeostatic plasticity (Borges et al., 2003; Fellin, 2009). Thus, the up-regulation of Kir4.1 associated with status epilepticusinduced astrogliosis might negatively regulate the TLE epileptogenesis by normalizing extracellular $\mathrm{K}^{+}\left(\left[\mathrm{K}^{+}\right]_{\mathrm{o}}\right)$ and glutamate $\left([\text { glutamate }]_{\mathrm{o}}\right)$. Furthermore, significantly higher Kir4.1 
expression ratios relative to the number of astrocytes (Kir4.1IR-positive cells/GFAP-IR-positive cells) were observed regionspecifically in the amygdaloid nuclei (i.e., MePV, MePD, and PMCo). These results illustrate the important role of amygdalar Kir4.1 channels in modifying status epileptics-induced epileptogenicity in TLE. Since deficit or knockdown of astrocytic Kir4.1 channels is known to impair $\mathrm{K}^{+}$- and glutamate-uptake into astrocytes and facilitate seizure generation (Djukic et al., 2007; Kucheryavykh et al., 2007; Bockenhauer et al., 2009; Scholl et al., 2009; Reichold et al., 2010; Sala-Rabanal et al., 2010; Tang et al., 2010), up-regulation of Kir4.1 channels in the pilocarpine TLE model seemed to occur as a compensatory mechanism to the limbic hyperexcitability in TLE epileptogenesis. Indeed, the medial amygdaloid and cortical amygdaloid nuclei are known to be closely linked to kindling epileptogenesis and human epileptic disorders including TLE (Hosford et al., 1995; Morimoto et al., 2004). Although it is known that pilocarpine-induced status epilepticus causes neural damage, sclerosis, and rewiring not only in the amygdala, but also in the Hpc, changes in the relative Kir4.1 expression ratios were not significant in the Hpc (i.e., CA2). This may be due to the relatively low expression level of Kir4.1 in the Hpc as compared to other brain regions (see Figure 2).

Evidence is accumulating that dysfunction of astrocytic Kir4.1 channels is causative of seizure activity generation. Specifically, loss-of-function mutations in human Kir4.1 gene (KCNJ10) cause the EAST syndrome, including GTC seizures and ataxia (Bockenhauer etal., 2009; Scholl etal., 2009; Reichold et al., 2010; Sala-Rabanal et al., 2010; Tang et al., 2010). It is also suggested that the down-regulation of Kir4.1 expression in the amygdala is related to seizure induction in an animal model of GTC seizures (Harada et al., 2013). Furthermore, recent studies showed the down-regulation and/or impaired functioning of Kir4.1 channels in specimens from patients with TLE (Das et al., 2012; Heuser et al., 2012; Steinhäuser et al., 2012), suggesting a close relationship of Kir4.1 to human TLE. The present results (Kir4.1 up-regulation) in the pilocarpine-induced TLE model, however, were different from the findings of Kir4.1 expression (Kir4.1 down-regulation) in patients with TLE. Although the reasons for this discrepancy are currently uncertain, it may result from the difference in the etiological basis between human TLE and pharmacologically evoked seizure. In fact, we also observed that Kir4.1 expression in the paralimbic cortex was gradually increased during the kindling development induced by pentylentetrazole (Mukai etal., 2013). Alternatively, it may be due to the temporal changes in Kir4.1 expression. Since the present study analyzed the Kir4.1 expression shortly after the occurrence of spontaneous seizures, the down-regulation of Kir4.1 may occur at a more advanced (delayed) stage in the pilocarpine-induced TLE model. Indeed, a recent study showed that Kir4.1 expression was down-regulated by local inflammatory events after TLE-associated brain injury, implying that the down-regulation of Kir4.1 could be a consequence, and not a primary cause, of seizures (Zurolo et al., 2012). Further studies are required to delineate the time course of the Kir4.1 expressional changes and the mechanisms underlying the Kir4.1 up-regulation in the pilocarpine-induced TLE model.
In conclusion, we performed expressional analysis of Kir4.1 in a pilocarpine-induced rat model of TLE to explore the pathophysiological role of Kir4.1 channels in epileptogenesis. Western blot analysis revealed that Kir4.1 levels of TLE rats under an interictal state were significantly increased in the cerebral cortex, St, and Ht while the levels of other Kir subunits, Kir5.1 and Kir2.1, were unaltered. Immunohistochemical analysis demonstrated that TLE rats showed a widespread elevation in Kir4.1 expression which accompanied an increase in the number of astrocytes per se. In addition, the Kir4.1 expression ratio relative to the increase in the astrocyte number was also elevated region-specifically in the amygdaloid nuclei in a pilocarpine TLE model. The present findings suggest that astrocytic Kir4.1 channels play a modulatory role in TLE epileptogenesis, possibly by acting as an inhibitory compensatory mechanism. Further studies using patch-clamp and/or microdialysis techniques are necessary to delineate the functional alterations (e.g., changes in Kir4.1-mediated potassium currents, extracellular levels of $\mathrm{K}^{+}$and glutamate) of up-regulated Kir4.1 channels in the TLE model.

\section{MATERIALS AND METHODS ANIMALS}

Male SD rats (7 weeks old; Japan SLC, Shizuoka, Japan) were used. Animals were kept in air-conditioned rooms under a 12-h light/dark cycle (light on: 6:00 AM) and allowed ad libitum access to food and water. The housing conditions of the rat and animal care methods complied with the NIH guide for the care and use of laboratory animals. The experimental protocols of this study were approved by the Experimental Animal Research Committee at Osaka University of Pharmaceutical Sciences.

\section{PILOCARPINE-INDUCED TLE MODEL}

A pilocarpine-induced TLE model was prepared according to methods reported previously (Cavalheiro, 1995; Liu et al., 2008). Briefly, animals were first treated with methyl-scopolamine (1 mg/kg, i.p., Sigma-Aldrich, St. Louis, MO, USA) to reduce peripheral cholinergic side effects and, 30 min later, pilocarpine $(350 \mathrm{mg} / \mathrm{kg}$, i.p., Sigma-Aldrich) was injected to induce acute status epilepticus. Pilocarpine-induced status epilepticus was then terminated by the injection of diazepam $(10 \mathrm{mg} / \mathrm{kg}$, i.p., CERCINE ${ }^{\circledR}$ INJECTION, Takeda Pharmaceutical Co. Ltd., Osaka, Japan) at 5, 20, 80, 300, and $420 \mathrm{~min}$ after the onset of status epilepticus (repeated and sustained clonic seizures). Animals which did not show any seizure activity (status epileptics) within $20 \mathrm{~min}$ after the pilocarpine injection were used as the control and treated with diazepam in the same manner as the status epilepticus-experienced rats. All animals were fed for 7-8 weeks after the pilocarpine treatment. Eleven out of the twelve rats which experienced pilocarpine-induced status epilepticus showed spontaneous seizures, (i.e., wild running/jumping and GTC seizures) and were defined as TLE rats. One animal which showed pilocarpine-induced status epilepticus but did not any spontaneous seizure was excluded from the analysis. None of the control animals $(N=11)$ showed any seizures or abnormal behavior during the 7-8 weeks observation period. 


\section{WESTERN BLOT ANALYSIS}

Temporal lobe epilepsy rats under interictal conditions $(N=4)$ or control rats $(N=4)$ were deeply anesthetized with pentobarbital $(80 \mathrm{mg} / \mathrm{kg}$, i.p.). The brain was then removed from the skull, chilled in ice-cold saline and dissected into the following 10 regions ( $\mathrm{fCx}, \mathrm{ptCx}$, otCx, St, Hpc, Th, Ht, Mid, $\mathrm{P} / \mathrm{MO}$, and Cer). Brain samples were then homogenized in an ice-cold lysis buffer ( $\mathrm{pH} 7.5$ ) containing: (in $\mathrm{mM}$ ) Tris 20, $\mathrm{NaCl} 150, \mathrm{MgCl}_{2}$ 10, EDTA 1.0, EGTA 1.0, 1\% Triton X100 , and a mixture of protease inhibitors (leupeptin, aprotinin, E-64, pepstatin A, bestatin, and 4-(2-aminoethyl) benzenesulfonyl fluoride hydrochloride; Nacalai Tesque, Kyoto, Japan). The homogenate was centrifuged at $15,000 \mathrm{~g}, 4^{\circ} \mathrm{C}$ for $30 \mathrm{~min}$ and the supernatant was stored at $-80^{\circ} \mathrm{C}$ for the Western blot analysis.

Western blots were performed as published previously (Ohno et al., 2009; Harada et al., 2013). Briefly, samples were incubated with a sodium dodecyl sulfate-polyacrylamide gel electrophoresis (SDS-PAGE) sample buffer for $5 \mathrm{~min}$ at $95^{\circ} \mathrm{C}$. Each sample (40 $\mu \mathrm{g} /$ lane) was then subjected to SDS-PAGE and separated proteins were transferred for $60 \mathrm{~min}$ to a PVDF membrane (GE Healthcare, Buckinghamshire, UK). The membrane was first incubated with a blocking solution containing $0.3-2 \%$ skim milk, $25 \mathrm{mM}$ Tris, $150 \mathrm{mM} \mathrm{NaCl}$, and $0.1 \%$ Tween $20(\mathrm{pH}$ 7.5) for $60 \mathrm{~min}$, then with the corresponding primary antibodies overnight $\left(4^{\circ} \mathrm{C}\right)$, followed by a 60 min-incubation with the secondary antibody, a goat anti-rabbit IgG-HRP conjugate (1:2000, Santa Cruz Biotechnology, CA, USA) for Kir4.1, a donkey anti-goat IgG-HRP conjugate (1:2000, Santa Cruz Biotechnology) for Kir5.1 or Kir2.1, or a sheep anti-mouse IgG-HRP conjugate (1:2000, GE Healthcare) for $\beta$-actin. The primary antibodies used were a rabbit polyclonal antibody against Kir4.1 (1:500, Alomone Labs., Jerusalem, Israel), a goat polyclonal antibody against Kir5.1 (N-12; 1:400, Santa Cruz Biotechnology), a goat polyclonal antibody against Kir2.1 (1:400, Santa Cruz Biotechnology) and mouse monoclonal antibodies against $\beta$-actin (1:1000, Sigma-Aldrich). Final detection was performed with the enhanced chemiluminescence methodology (Amersham ECL Western blotting detection reagents and analysis system, GE Healthcare) using a lumino imaging analyzer (LAS-3000, FUJIFILM, Tokyo, Japan). To normalize for protein loading, chemiluminescence of the bands in each lane was standardized to the intensity of the $\beta$-actin band in the same lane.

\section{IMMUNOHISTOCHEMICAL ANALYSIS}

Brains were obtained from TLE rats (interictal status; $N=7$ ) or control rats $(N=7)$ in the same manner as for the Western

\section{REFERENCES}

Amiry-Moghaddam, M., and Ottersen, O. P. (2003). The molecular basis of water transport in the brain. Nat. Rev. Neurosci. 4, 991-1001. doi: 10.1038/nrn 1252

Bartolomei, F., Khalil, M., Wendling, F., Sontheimer, A., Régis, J., Ranjeva, J. P., et al. (2005). Entorhinal cortex involvement in human mesial temporal lobe epilepsy: an electrophysiologic and volumetric study. Epilepsia 46, 677687. doi: 10.1111/j.1528-1167.2005. 43804.x

Bockenhauer, D., Feather, S., Stanescu, H. C., Bandulik, S., Zdebik, A. A. Reichold, M., et al. (2009). Epilepsy, ataxia, sensorineural deafness, tubulopathy, and KCNJ10 mutations. $N$.

blot analysis. After fixation in a $4 \%$ paraformaldehyde solution for $24 \mathrm{~h}$, brain samples were dehydrated and embedded in paraffin. Formalin-fixed and paraffin-embedded tissue samples were cut into $4-\mu \mathrm{m}$ thick sections and a pair of successive slices in each brain region was immunohistochemically stained with anti-Kir4.1 or anti-GFAP antibody using the avidin-biotin complex (ABC) method (Ohno et al., 2009, 2012; Harada et al., 2013). Briefly, the fronto- and occipito-temporal brain sections were deparaffinized in xylene and then rehydrated in ethanol. Sections were autoclaved for $10 \mathrm{~min}$ to retrieve the antigen. After cooling to room temperature, endogenous peroxidase activity was quenched by $3 \% \mathrm{H}_{2} \mathrm{O}_{2}$ and non-specific binding was blocked using a $5 \%$ skim milk solution. Sections were then incubated overnight $\left(4^{\circ} \mathrm{C}\right)$ with a rabbit anti-Kir4.1 antibody $(1: 100$, Alomone Labs) and a mouse anti-GFAP antibody (1:100, Progen) in the 5\% skim milk solution. Thereafter, they were incubated with a biotinylated goat anti-rabbit IgG secondary antibody (1:400, Vector Laboratories, Burlingame, CA, USA) and a goat antimouse IgG secondary antibody (1:400, Sigma-Aldrich) for $60 \mathrm{~min}$ and with an avidin-biotinylated horseradish peroxidase complex (Vectastain ABC Kit) for an additional $60 \mathrm{~min}$. Kir4.1- and GFAP-IR was visualized by the diaminobenzidine-nickel staining method.

The number of Kir4.1- or GFAP-IR-positive cells was counted in a $350 \times 350 \mu \mathrm{m}^{2}$ grid laid over various regions of the brain (Figure 4), which included the following regions: the motor cortex (MC), SC, AID, ectorhinal-perirhinal cortex (Ect-PRh), Pir, dorsolateral St (dlST) and dmST, vlST and ventromedial St (vmST), core (AcbC) and shell (AcbSh) regions of the nucleus accumbens, MePV, MePD, basolateral amygdaloid nucleus posterior part (BLP), basomedial amygdaloid nucleus posterior part (BMP), PMCo, and CA1, CA3, and the DG of the Hpc. Relative expression rate of Kir4.1 was defined as a percentage of the number of Kir4.1-positive cells relative to that of GFAP-positive cells.

\section{STATISTICAL ANALYSIS}

All data are expressed as the mean \pm SEM. Expressional changes in Kir channel subunits determined by Western blot or immunohistochemical analysis were compared by two-way ANOVA followed by Tukey multiple comparison test. Differences were considered to be statistically significant for values of $P<0.05$.

\section{ACKNOWLEDGMENTS}

This work was partly supported by a Grant in Aid for Scientific Research (22590092) from the Ministry of Education, Science, Sports and Culture of Japan and a research grant from the Japan Epilepsy Research Foundation.

Engl. J. Med. 360, 1960-1970. doi: 10.1056/NEJMoa0810276

Borges, K., Gearing, M., McDermott,

D. L., Smith, A. B., Almonte, A. G., Wainer, B. H., et al. (2003). Neuronal and glial pathological changes during epileptogenesis in the mouse pilocarpine model. Exp. Neurol. 182, 21-34. doi: 10.1016/S00144886(03)00086-4
Butt, A. M., and Kalsi, A. (2006). Inwardly rectifying potassium channels (Kir) in central nervous system glia: a special role for Kir4.1 in glial functions. J. Cell. Mol. Med. 10, 3344. doi: 10.1111/j.1582-4934.2006. tb00289.x

Cavalheiro, E. A. (1995). The pilocarpine model of epilepsy. 
Ital. J. Neurol. Sci. 16, 33-37. doi: 10.1007/BF02229072

Cavalheiro, E. A., Leite, J. P., Bortolotto, Z. A., Turski, W. A., Ikonomidou, C., and Turski, L. (1991). Long-term effects of pilocarpine in rats: structural damage of the brain triggers kindling and spontaneous recurrent seizures. Epilepsia 32, 778782. doi: 10.1111/j.1528-1157.1991. tb05533.x

Chakir, A., Fabene, P. F., Ouazzani, R., and Bentivoglio, M. (2006). Drug resistance and hippocampal damage after delayed treatment of pilocarpine-induced epilepsy in the rat. Brain Res. Bull. 71, 127138. doi: 10.1016/j.brainresbull.2006. 08.009

Connors, N. C., Adams, M. E., Froehner, S. C., and Kofuji, P. (2004). The potassium channel Kir4.1 associates with the dystrophin-glycoprotein complex via alpha-syntrophin in glia. $J$. Biol. Chem. 279, 28387-28392. doi: 10.1074/jbc.M402604200

Curia, G., Longo, D., Biagini, G., Jones, R. S., and Avoli, M. (2008). The pilocarpine model of temporal lobe epilepsy. J. Neurosci. Methods 172, 143-157. doi: 10.1016/j.jneumeth.2008.04.019

Das, A., Wallace, G. C., Holmes, C., McDowell, M. L., Smith, J. A., Marshall, J. D., et al. (2012). Hippocampal tissue of patients with refractory temporal lobe epilepsy is associated with astrocyte activation, inflammation, and altered expression of channels and receptors. Neuroscience 220, 237-246. doi: 10.1016/j.neuroscience.2012. 06.002

Djukic, B., Casper, K. B., Philpot, B. D., Chin, L. S., and McCarthy, K. D. (2007). Conditional knockout of Kir4.1 leads to glial membrane depolarization, inhibition of potassium and glutamate uptake, and enhanced short-term synaptic potentiation. J. Neurosci. 27, 11354 11365. doi: 10.1523/JNEUROSCI. 0723-07.2007

Fellin, T. (2009). Communication between neurons and astrocytes: relevance to the modulation of synaptic and network activity. J. Neurochem. 108, 533-544. doi: 10.1111/j.14714159.2008.05830.x

Furutani, K., Ohno, Y., Inanobe, A., Hibino, H., and Kurachi, Y. (2009). Mutational and in silico analyses for antidepressant block of astroglial inward-rectifier Kir4.1 channel. Mol. Pharmacol. 75, 1287-1295. doi: 10.1124/mol.108.052936

Glien, M., Brandt, C., Potschka, H., and Löscher, W. (2002). Effects of the novel antiepileptic drug levetiracetam on spontaneous recurrent seizures in the rat pilocarpine model of temporal lobe epilepsy. Epilepsia 43, 350-357. doi: 10.1046/j.15281157.2002.18101.X

Harada, Y., Nagao, Y., Shimizu, S. Serikawa, T., Terada, R., Fujimoto, M., et al. (2013). Expressional analysis of inwardly rectifying Kir4.1 channels in Noda epileptic rat (NER). Brain Res. 1517, 141149. doi: 10.1016/j.brainres.2013. 04.009

Hauser, W. A., Annegers, J. F., and Rocca, W. A. (1996). Descriptive epidemiology of epilepsy: contributions of population-based studies from Rochester, Minnesota. Mayo Clin. Proc. 71, 576-586. doi: 10.4065/71.6.576

Heuser, K., Eid, T., Lauritzen, F., Thoren, A. E., Vindedal, G. F. Taubøll, E., et al. (2012). Loss of perivascular Kir4.1 potassium channels in the sclerotic hippocampus of patients with mesial temporal lobe epilepsy. J. Neuropathol. Exp. Neurol. 71, 814-825. doi: 10.1097/ NEN.0b013e318267b5af

Heuser, K., Nagelhus, E. A., Taubøll, E., Indahl, U., Berg, P. R., Lien, S., et al. (2010). Variants of the genes encoding AQP4 and Kir4.1 are associated with subgroups of patients with temporal lobe epilepsy. Epilepsy Res. 88, 5564. doi: 10.1016/j.eplepsyres.2009. 09.023

Hibino, H., Fujita, A., Iwai, K., Yamada, M., and Kurachi, Y. (2004). Differential assembly of inwardly rectifying $\mathrm{K}+$ channels subunits, Kir4.1 and Kir5.1, in brain astrocytes. J. Biol. Chem. 279, 44065-44073. doi: 10.1074/jbc.M405985200

Hosford, D. A., Simonato, M., Cao, Z., Garcia-Cairasco, N., Silver, J. M., Butler, L., et al. (1995). Differences in the anatomic distribution of immediate-early gene expression in amygdala and angular bundle kindling development. J. Neurosci. 15, 2513-2523.

Howe, M. W., Feig, S. L., Osting, S. M., and Haberly, L. B. (2008). Cellular and subcellular localization of Kir2.1 subunits in neurons and glia in piriform cortex with implications for $\mathrm{K}+$ spatial buffering. $J$. Comp. Neurol. 506, 877-893. doi: 10.1002/cne. 21534

Kang, S. J., Cho, S. H., Park, K., Yi, J., Yoo, S. J., and Shin, K. S. (2008). Expression of Kir2.1 channels in astrocytes under pathophysiological conditions. Mol. Cells 25, 124-130.
Kofuji, P., and Newman, E. A. (2004). Potassium buffering in the central nervous system. Neuroscience 129, 1045-1056. doi: 10.1016/j.neuroscience.2004.06.008

Kucheryavykh, Y. V., Kucheryavykh, L. Y., Nichols, C. G., Maldonado, H. M., Baksi, K., Reichenbach, A., et al. (2007). Downregulation of Kir4.1 inward rectifying potassium channel subunits by RNAi impairs potassium transfer and glutamate uptake by cultured cortical astrocytes. Glia 55, 274-281. doi: 10.1002/glia. 20455

Leite, J. P., Bortolotto, Z. A., and Cavalheiro, E. A. (1990). Spontaneous recurrent seizures in rats: an experimental model of partial epilepsy. Neurosci. Biobehav. Rev. 14, 511517. doi: 10.1016/S0149-7634(05) 80076-4

Liu, X. Y., Yang, J. L., Chen, L. J., Zhang, Y., Yang, M. L., Wu, Y. Y., et al. (2008). Comparative proteomics and correlated signaling network of rat hippocampus in the pilocarpine model of temporal lobe epilepsy. Proteomics 8, 582-603. doi: 10.1002/pmic. 200700514

Löscher, W. (2002). Animal models of epilepsy for the development of antiepileptogenic and diseasemodifying drugs. A comparison of the pharmacology of kindling and post-status epilepticus models of temporal lobe epilepsy. Epilepsy Res. 50, 105-123.

Maldonado, P. P., Vélez-Fort, M., Levavasseur, F., and Angulo, M. C. (2013). Oligodendrocyte precursor cells are accurate sensors of local $\mathrm{K}+$ in mature gray matter. $\mathrm{J}$. Neu rosci. 33, 2432-2442. doi: 10.1523/ JNEUROSCI.1961-12.2013

Mathern, G. W., Kuhlman, P. A., Mendoza, D., and Pretorius, J. K. (1997). Human fascia dentata anatomy and hippocampal neuron densities differ depending on the epileptic syndrome and age at first seizure. J. Neuropathol. Exp. Neurol. 56, 199-212. doi: 10.1097/00005072-19970200000011

Morimoto, K., Fahnestock, M., and Racine, R. J. (2004). Kindling and status epilepticus models of epilepsy: rewiring the brain. Prog. Neurobiol. 73, 1-60. doi: 10.1016/j.pneurobio.2004.03.009

Mukai, T., Nagao, Y., Ono, A., Sakagami, Y., Okuda, A., Fujimoto, M., et al. (2013). Expression analysis of astrocytic Kir4.1 channels in pentylenetetrazol-induced kindling model. J. Pharmacol. Sci. 121(Suppl. 1), 216P. doi: 10.1016/ j.jns.2010.06.002
Nagelhus, E. A., Horio, Y., Inanobe, A., Fujita, A., Haug, F. M., Nielsen, S., et al. (1999). Immunogold evidence suggests that coupling of $\mathrm{K}+$ siphoning and water transport in rat retinal Müller cells is mediated by a coenrichment of Kir4.1 and AQP4 in specific membrane domains. Glia 26, 47-54. doi: 10.1002/(SICI)10981136(199903)26:1

Ohno, Y., Hibino, H., Lossin, C., Inanobe, A., and Kurachi, Y. (2007). Inhibition of astroglial Kir4.1 channels by selective serotonin reuptake inhibitors. Brain Res. 1178, 44-51. doi: 10.1016/j.brainres.2007. 08.018

Ohno, Y., Ishihara, S., Terada, R., Kikuta, M., Sofue, N., Kawai, Y., et al. (2009). Preferential increase in the hippocampal synaptic vesicle protein 2A (SV2A) by pentylenetetrazole kindling. Biochem. Biophys. Res. Commun. 390, 415-420. doi: 10.1016/j.bbrc.2009.09.035

Ohno, Y., Okumura, T., Terada, R., Ishihara, S., Serikawa, T., and Sasa, M. (2012). Kindling-associated SV2A expression in hilar GABAergic interneurons of the mouse dentate gyrus. Neurosci. Lett. 510, 93-98. doi: 10.1016/j.neulet.2012.01.009

Puwarawuttipanit, W., Bragg, A. D., Frydenlund, D. S., Mylonakou, M. N., Nagelhus, E. A., Peters, M. F, et al. (2006). Differential effect of alpha-syntrophin knockout on aquaporin-4 and Kir4.1 expression in retinal macroglial cells in mice. Neuroscience 137, 165-175. doi: 10.1016/j.neuroscience.2005. 08.051

Reichold, M., Zdebik, A. A., Lieberer, E., Rapedius, M., Schmidt, K., Bandulik, S., et al. (2010). KCNJ10 gene mutations causing EAST syndrome (epilepsy, ataxia, sensorineural deafness, and tubulopathy) disrupt channel function. Proc. Natl. Acad. Sci. U.S.A. 107, 14490-14495. doi: 10.1073/pnas.1003072107

Sala-Rabanal, M., Kucheryavykh, L. Y., Skatchkov, S. N., Eaton, M. J., and Nichols, C. G. (2010). Molecular mechanisms of EAST/SeSAME syndrome mutations in Kir4.1 (KCNJ10). J. Biol. Chem. 285, 36040-36048. doi: 10.1074/jbc. M110.163170

Scholl, U. I., Choi, M., Liu, T., Ramaekers, V. T., Häusler, M. G., Grimmer, J., et al. (2009). Seizures, sensorineural deafness, ataxia, mental retardation, and electrolyte imbalance (SeSAME syndrome) caused by mutations in KCNJ10. Proc. Natl. Acad. Sci. U.S.A. 106, 5842-5847. doi: 10.1073/pnas.0901749106 
Seifert, G., Hüttmann, K., Binder, D. K., Hartmann, C., Wyczynski, A., Neusch, C., et al. (2009). Analysis of astroglial $\mathrm{K}+$ channel expression in the developing hippocampus reveals a predominant role of the Kir4.1 subunit. J. Neurosci. 29, 7474-7488. doi: 10.1523/JNEUROSCI.3790-08.2009

Simard, M., and Nedergaard, M. (2004). The neurobiology of glia in the context of water and ion homeostasis. Neuroscience 129, 877896. doi: 10.1016/j.neuroscience. 2004.09.053

Steinhäuser, C., Seifert, G., and Bedner, P. (2012). Astrocyte dysfunction in temporal lobe epilepsy: $\mathrm{K}+$ channels and gap junction coupling. Glia 60, 1192-1202. doi: 10.1002/glia. 22313

Su, S., Ohno, Y., Lossin, C., Hibino, H., Inanobe, A., and Kurachi,
Y. (2007). Inhibition of astroglial inwardly rectifying Kir4.1 channels by a tricyclic antidepressant, nortriptyline. J. Pharmacol. Exp. Ther. 320, 573-580. doi: 10.1124/jpet.106. 112094

Tanemoto, M., Kittaka, N., Inanobe, A. and Kurachi, Y. (2000). In vivo formation of a proton-sensitive $\mathrm{K}+$ channel by heteromeric subunit assembly of Kir5.1 with Kir4.1. J. Physiol. 525, 587-592. doi: 10.1111/j.14697793.2000.00587.x

Tang, X., Hang, D., Sand, A., and Kofuji, P. (2010). Variable loss of Kir4.1 channel function in SeSAME syndrome mutations. Biochem. Biophys. Res. Commun. 399, 537-541. doi: 10.1016/j.bbrc.2010.07.105

Walz, W. (2000). Role of astrocytes in the clearance of excess extracellular potassium. Neurochem. Int.
36, 291-300. doi: 10.1016/S01970186(99)00137-0

Wieser, H. G. (2004). ILAE Commission Report. Mesial temporal lobe epilepsy with hippocampal sclerosis. Epilepsia 45, 695-714. doi: 10.1111/j.0013-9580.2004.09004.x

Zurolo, E., de, Groot, M., Iyer, A. Anink, J., van Vliet, E. A., Heimans, J. J., et al. (2012). Regulation of Kir4.1 expression in astrocytes and astrocytic tumors: a role for interleukin1 ß. J. Neuroinflammation 9, 280. doi:10.1186/1742-2094-9-280

Conflict of Interest Statement: The authors declare that the research was conducted in the absence of any commercial or financial relationships that could be construed as a potential conflict of interest.
Received: 30 April 2013; accepted: 16 June 2013; published online: 05 July 2013.

Citation: Nagao Y, Harada Y, Mukai T, Shimizu S, Okuda A, Fujimoto M, Ono A, Sakagami Y and Ohno Y (2013) Expressional analysis of the astrocytic Kir4.1 channel in a pilocarpine-induced temporal lobe epilepsy model. Front. Cell. Neurosci. 7:104. doi: 10.3389/fncel.2013. 00104

Copyright (c) 2013 Nagao, Harada, Mukai, Shimizu, Okuda, Fujimoto, Ono, Sakagami and Ohno. This is an openaccess article distributed under the terms of the Creative Commons Attribution License, which permits use, distribution and reproduction in other forums, provided the original authors and source are credited and subject to any copyright notices concerning any third-party graphics etc. 


\section{APPENDIX}
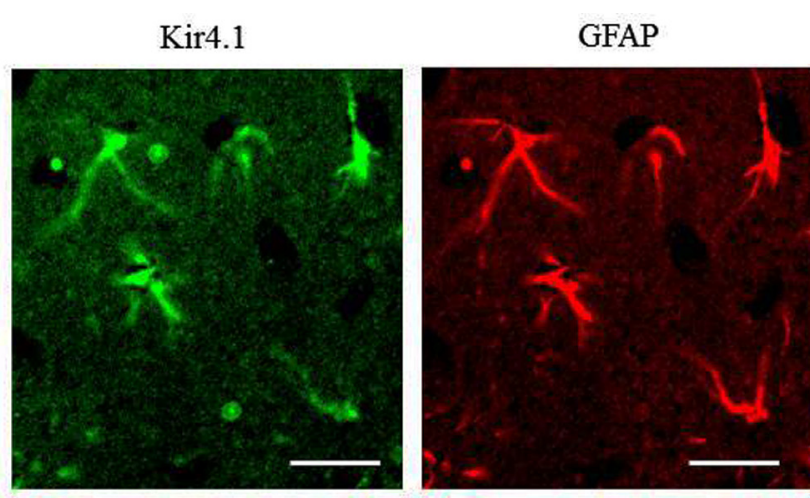

Merged (Kir4.1/GFAP)

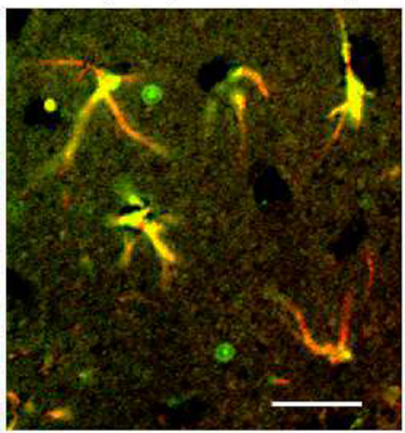

FIGURE A1 | Expressional patterns of Kir4.1 in the rat hippocampus. Typical photograph illustrating a double staining of Kir4.1 with GFAP in the hippocampal CA1 field. The hippocampal section was incubated anti-GFAP antibody (Progen, Heidelberg, Germany) for $24 \mathrm{~h}$ at $4^{\circ} \mathrm{C}$ and then incubated with a FITC (fluorescein isothiocyanate; green fluorescence)-conjugated goat anti-rabbit IgG secondary antibody (Sigma-Aldrich) or TRITC (tetramethylrhodamine-5- (and 6)-isothiocyanate; red fluorescence)-conjugated goat anti-mouse IgG secondary antibody
(Sigma-Aldrich) to probe Kir4.1 and GFAP, respectively. Immunofluorescence images were obtained with a confocal laser scanning microscope. Scale bar: $50 \mu \mathrm{m}$. All the Kir4.1-immunoreactivity (IR)-positive cells with the stellate-shape were double-stained with anti-GFAP antibody (yellow in a merged picture). Kir4.1-IR was occasionally found in a few round-shaped cells with no GFAP-IR [also negative to a neuronal marker, neuronal nuclear antigen (NeuN)], which were omitted from the analysis. 